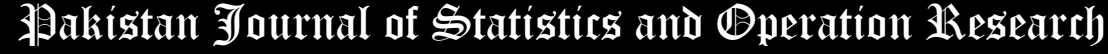

\section{On Erlang-Truncated Exponential Distribution: Theory and Application}

\author{
I. Elbatal ${ }^{1 *}$, A. Aldukeel ${ }^{2}$ \\ * Corresponding Author \\ 1. Department of Mathematics and Statistics-College of Science-Imam Mohammad Ibn Saud Islamic University \\ (IMSIU)- Saudi Arabia. iielbatal@imamu.edu.sa \\ 2. Department of Mathematics and Statistics-College of Science-Imam Mohammad Ibn Saud Islamic University \\ (IMSIU)- Saudi Arabia. amdukhil@imamu.edu.sa
}

\begin{abstract}
In this article, we introduce a new distribution called the McDonald Erlang-truncated exponential distribution. Various structural properties including explicit expressions for the moments, moment generating function, mean deviation of the new distribution are derived. The estimation of the model parameters is performed by maximum likelihood method. The usefulness of the new distribution is illustrated by two real data sets. The new model is much better than other important competitive models in modeling relief times and survival times data sets.
\end{abstract}

Key Words: Erlang-truncated Exponential distribution, McDonald distribution, moments, Moment generating function, Maximum Likelihood Estimation.

Mathematical Subject Classification: 62N02; 62N01; 62E10.

\section{Introduction and motivation}

The Erlang-truncated exponential distribution (ETEx) is widely used in the field of queuing system and stochastic processes. El-Alosey (2007) introduced Erlang-truncated exponential (ETEx) distribution by mixing the Erlang distribution with left truncated one parameter exponential distribution. The ETEx distribution, like the exponential distribution, has a constant failure rate which makes it practically impossible for the model to provide a reasonable parametric fit to data sets with decreasing failure rate, increasing failure rate and non-monotonic failure rate such as the bathtub and unimodal failure rates which are common in reliability studies and other related fields of studies. The cumulative distribution function (CDF) of this distribution is given by

$$
G_{\mathrm{ETEx}}^{(\alpha, \theta)}(x)=1-\left.e^{-\alpha\left(1-e^{-\theta}\right) x}\right|_{(x \geq 0)},
$$

where $\theta>0$ is the scale parameter and $\alpha>0$ is the shape parameter. When $\theta \rightarrow \infty$, we have the standard exponential model. The corresponding probability density function (PDF) and hazard rate function (HRF) are given by

and

$$
g_{\mathrm{ETEx}}^{(\alpha, \theta)}(x)=\left.\alpha\left(1-e^{-\theta}\right) e^{-\alpha\left(1-e^{-\theta}\right) x}\right|_{(x \geq 0)},
$$

$$
h_{\mathrm{ETEx}}^{(\alpha, \theta)}(x)=\alpha\left(1-e^{-\theta}\right)
$$

respectively. As illustrated in (3), the HRF of the ETEx distribution is constant and this which makes it inadequate for modelling many complex lifetime data sets that have nonconstant failure rates. So, the main aim of this paper is to extend the ETEx distribution by adding three extra shape parameters to define a new flexible model referred to as the Mc-Donald Erlang-truncated exponential (McETEx) distribution. The role of the three additional parameters is to introduce skewness and to vary tail weights and provide greater flexibility in the shape of the generalized distribution and consequently in modeling observed data. It may be mentioned that although several skewed distribution functions exist on the positive real axis not many skewed distributions are available on the whole real line which are easy to use 
for data analysis purpose. The main idea is to introduce three shape parameters, so that the McETEx distribution can be used to model skewed data, a feature which is very common in practice.

The PDF of the Mc-Donald G family (Mc-G) is defined by

$$
f_{\mathrm{Mc}-\mathrm{G}}^{(a, b, c)}(x ; \varphi)=\frac{c}{B(a, b)} g(x ; \varphi) G^{a c-1}(x ; \varphi)\left[1-G^{c}(x ; \varphi)\right]^{b-1},
$$

where $a>0, b>0$ and $c>0$ are additional shape parameters. Note that $g(x ; \varphi)$ is the PDF of baseline distribution, $g(x ; \varphi)=d G(x ; \varphi) / d x$ and $\varphi$ is the paramrt vector. The class of distributions (1.4) includes as special sub-models the beta generalized (B-G) family of distributions for $c=1$ (see Eugene et al. (2002)) and Kumaraswamy generalized (K-G) family of distributions (see Cordeiro and Castro (2011)) for $a=1$. The corresponding CDF OF (4) is given by

where

$$
F_{\mathrm{Mc}-\mathrm{G}}^{(a, b, c)}(x ; \varphi)=I_{G^{c}(x ; \varphi)}(a, b)=\frac{1}{B(a, b)} \int_{0}^{G(x ; \varphi)^{c}} \omega^{a-1}(1-\omega)^{b-1} d \omega,
$$

$$
I_{\zeta}(a, b)=\frac{1}{B(a, b)} \int_{0}^{\zeta} \omega^{a-1}(1-\omega)^{b-1} d \omega,
$$

denotes the incomplete beta function ratio (Gradshteyn and Ryzhik, (2000)). Equation (5) can also be rewritten as follows

$$
F_{\mathrm{Mc}-\mathrm{G}}^{(a, b, c)}(x ; \varphi)=\frac{G(x ; \varphi)^{a c}}{a B(a, b)}{ }_{2} F_{1}\left(a, 1-b ; a+1 ; G(x ; \varphi)^{c}\right),
$$

where

$$
2 F_{1}(a, b ; c ; x)=B(b, c-b)^{-1} \int_{0}^{1} \frac{t^{b-1}(1-t)^{c-b-1}}{(1-t x)^{a}} d t,
$$

is the well-known "hypergeometric function" which are well established in the literature (see Gradshteyn and Ryzhik (2000)). The HRF and reverse hazard functions (RHF) of the Mc-G distribution are given by

$$
h_{\mathrm{Mc}-\mathrm{G}}^{(a, b, c)}(x ; \varphi)=\frac{c g(x ; \varphi) G^{a c-1}(x ; \varphi)\left[1-G^{c}(x ; \varphi)\right]^{b-1}}{B(a, b)\left[1-I_{G^{c}(x ; \varphi)}(a, b)\right]},
$$

and

$$
\tau_{\mathrm{Mc}-\mathrm{G}}^{(a, b, c)}(x ; \varphi)=\frac{c g(x ; \varphi) G^{a c-1}(x ; \varphi)\left[1-G^{c}(x ; \varphi)\right]^{b-1}}{B(a, b)\left[I_{G^{c}(x ; \varphi)}(a, b)\right]}
$$

respectively. Recently, many authors used the Mc-G family to expand some existing models such as the McDonald gamma distribution by Marciano et al. (2012), the McDonald Normal distribution by Cordeiro et al. (2012), McDonald half-logistic distribution by Oliveira et al. (2013), McDonald Dagum by Oluyede and Rajasooriya (2013), the McDonald Gompertz distribution by Roozegar et al. (2017).

The rest of the article is organized as follows. In Section 2, we define the McETEx distribution. The expansion for the cumulative and density functions of the (McETEx) distribution and some special cases are proposed in Section 2. Moments, moment generating function and conditional moment. Mean deviation, Lorenz and Bonferroni curves are discussed in Section 3. In Section 4 Residual life and Reversed Failure Rate Function of (McETEx) distribution. Maximum likelihood estimation is performed in Section 5. In Section 6, we provide application to real data set to illustrate the importance of the new distribution.

\section{The new distribution}

Using (1) and (5) the CDF of (McETEx) can be written as

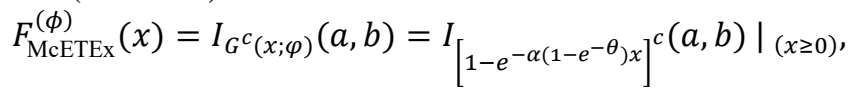

the corresponding PDF is given by 


$$
f_{\text {McETEx }}^{(\phi)}(x)=\frac{c \alpha\left(1-e^{-\theta}\right)}{B(a, b)} e^{-\alpha\left(1-e^{-\theta}\right) x}\left[1-e^{-\alpha\left(1-e^{-\theta}\right) x}\right]^{a c-1}\left\{1-\left[1-e^{-\alpha\left(1-e^{-\theta}\right) x}\right]^{c}\right\}^{b-1} \mathrm{I}_{(x \geq 0)} .
$$

The new distribution will attract wider applications in reliability as well as in other areas of research and it can be used in a variety of problems in modeling survival data (see Section 7). Figure 1 represents some plots of the probability density function of the McETEx distribution for some different parameter values. Table 1 gives some sub models from the McETEx model.

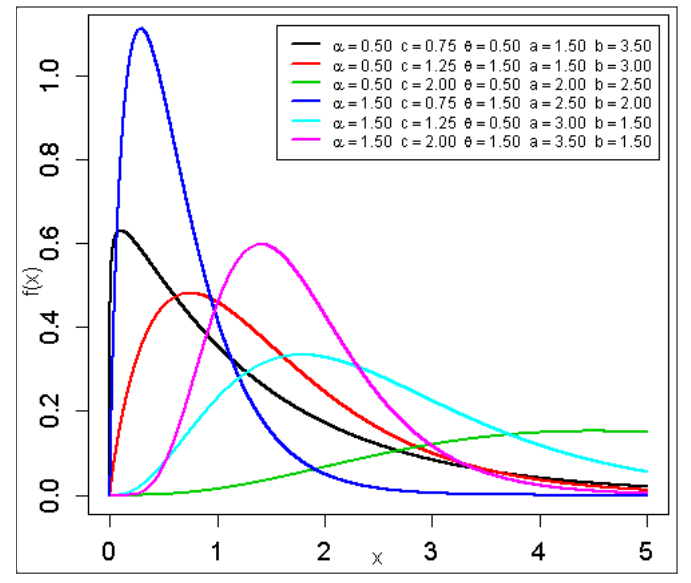

Figure 1: Plots of the PDF of McETEx for some different parameter values.

Table 1: Some sub models from the McETEx model.

\begin{tabular}{cccccc}
\hline$a$ & $b$ & $c$ & $\alpha$ & $\theta$ & Reduced model \\
\hline$a$ & $b$ & $c$ & $\alpha$ & $\rightarrow \infty$ & McEx \\
$a$ & $b$ & 1 & $\alpha$ & $\theta$ & BETEx \\
$a$ & $b$ & 1 & $\alpha$ & $\rightarrow \infty$ & BEx \\
1 & $b$ & $c$ & $\alpha$ & $\theta$ & KETEx \\
1 & $b$ & $c$ & $\alpha$ & $\rightarrow \infty$ & KEx \\
\hline
\end{tabular}

A random variable $X$ with the PDF (9) is said to have a McETEx distribution and will be denoted by $X \sim$ McETEx $(\phi)$ where $\phi=(a, b, c, \alpha, \theta)$. The HRFcan be defined as

$$
h_{\text {MCETEx }}(x, \phi)=\frac{c \alpha\left(1-e^{-\theta}\right) e^{-\alpha\left(1-e^{-\theta}\right) x}\left[1-\left(1-e^{-\alpha\left(1-e^{-\theta}\right) x}\right)^{c}\right]^{b-1}}{B(a, b)\left[1-I_{\left(1-e^{-\alpha\left(1-e^{-\theta}\right) x}\right) c}(a, b)\right]\left[1-e^{-\alpha\left(1-e^{-\theta}\right) x}\right]^{-a c+1}} .
$$

Many extension of the ETEx model can be cited such as Okorie et al. (2016) introduced the transmuted Erlangtruncated exponential distribution, Nasiru et al. (2016) investigated the generalized Erlang-truncated exponential distribution and Okorie et al. (2017) proposed and studied the Marshall-Olkin generalized Erlang-truncated exponential distribution, among others. Many other useful extensions can found in Alizadeh et al. (2018), Hamedani et al. (2018), Elbiely and Yousof (2019), Alizadeh et al. (2020), Ibrahim et al. (2020), Karamikabir et al. (2020), Yousof et al. (2018a,b,c, 2019 and 2020), Korkmaz et al. (2018, 2019a,b,c and 2020) and Ibrahim et al. (2020).

\section{Useful Expansions}

In this Section, we present some representations of PDF of McETEx distribution. The mathematical relation given below will be useful in this subsection. The series representation given below will be useful in this subsection. If $\zeta$ is a positive real non- integer and $|\tau|<1$ then

$$
(1-\tau)^{\zeta}=\sum_{k=0}^{\infty}(-\tau)^{k}\left(\begin{array}{l}
\zeta \\
k
\end{array}\right)
$$

Substituting from (11) into (9), we get 


$$
f_{\text {McETEx }}^{(\phi)}(x)=\frac{c \alpha\left(1-e^{-\theta}\right)}{B(a, b)} \sum_{i=0}^{\infty}(-1)^{i}\left(\begin{array}{c}
b-1 \\
i
\end{array}\right) e^{-\alpha\left(1-e^{-\theta}\right) x}\left[1-e^{-\alpha\left(1-e^{-\theta}\right) x}\right]^{c(a+i)-1},
$$

again, using the binomial series expansion in (12) we get

$$
f_{\text {McETEx }}^{(\phi)}(x)=\sum_{i, j=0}^{\infty} \xi_{i, j} e^{-\vartheta x},
$$

where

and

$$
\xi_{i, j}=c \alpha\left(1-e^{-\theta}\right) \frac{(-1)^{i+j}}{B(a, b)}\left(\begin{array}{c}
b-1 \\
i
\end{array}\right)\left(\begin{array}{c}
c(a+i)-1 \\
j
\end{array}\right),
$$

$$
\vartheta=\alpha(j+1)\left(1-e^{-\theta}\right) .
$$

\section{Statistical Properties Moments}

The following theorems give the rth moment $\left(\mu_{r}\right)$ and moment generating function $M_{X}(t)$ of the distribution McETEx.

Theorem (4.1): If $X$ has the McETEx, then the rth moment of $X$ is given by the following

where

$$
\mu_{r}^{\prime}=\Gamma(1+r) \sum_{i, j=0}^{\infty} \xi_{i, j}^{(1+r, \vartheta)}
$$

and

$$
\xi_{i, j}^{(1+r, \vartheta)}=\frac{1}{\vartheta^{1+r}} \xi_{i, j}
$$

Further, the central moments $\mu_{r}$ and cumulants $\kappa_{r}$ of the McETEx distribution can be determined by

$$
\mu_{r}=\sum_{m=0}^{r}\left(\begin{array}{c}
r \\
m
\end{array}\right)(-1)^{m} \mu_{1}^{\prime m} \mu_{r-m}^{\prime}
$$

respectively, where

$$
\kappa_{r}=\mu_{r}^{\prime}-\sum_{m=1}^{r-1} \kappa_{m}\left(\begin{array}{c}
r-1 \\
m-1
\end{array}\right) \mu_{r-m}^{\prime}
$$

and

$$
\kappa_{1}=\mu_{1}^{\prime}, \kappa_{2}=\mu_{2}^{\prime}-\mu_{1}^{/ 2}, \kappa_{3}=\mu_{3}^{\prime}-3 \mu_{2}^{\prime} \mu_{1}^{\prime}+2 \mu_{1}^{/ 3},
$$

$$
\kappa_{4}=\mu_{4}^{\prime}-4 \mu_{1}^{\prime} \mu_{3}^{\prime}-3 \mu_{2}^{\prime 2}+12 \mu_{2}^{\prime} \mu_{1}^{/ 2}-6 \mu_{1}^{/ 4} .
$$

The coefficient of variation (CV), coefficient of skewness (CS) and coefficient of kurtosis (CK) can be obtained according to the following relation

and

$$
C V=\sqrt{\frac{\mu_{2}^{\prime}}{\mu_{2}}-1}, C S=\frac{\kappa_{3}}{\sqrt{\kappa_{2}^{3}}}
$$

$$
C K=\frac{\kappa_{4}}{\kappa_{2}^{2}}
$$

respectively.

Theorem (4.2): If $X$ has the McETEx ( $\phi$ ), then the the moment generating function (MGF) of $X$ is given as follows

\section{Conditional moments}

$$
M_{X}(t)=\Gamma(1+r) \sum_{i, j=0}^{\infty} \frac{\xi_{i, j}}{\vartheta-t} .
$$

For lifetime models, it is of interest to know the rth lower and upper incomplete moments of $X$ defined by 
and

$$
v_{S}(t)=E\left(\left.X^{s}\right|_{(X<t)}\right)=\int_{0}^{t} x^{s} f(x) d x
$$

$$
\eta_{s}(t)=E\left(\left.X^{s}\right|_{(X>t)}\right)=\int_{t}^{\infty} x^{s} f(x) d x
$$

respectively, for any real $s>0$. The $\mathrm{r}$ th lower incomplete moment of McETEx distribution is

where

$$
v_{s}(t)=\int_{0}^{t} x^{s} f(x) d x=\sum_{i, j=0}^{\infty} \xi_{i, j} \int_{0}^{t} x^{s} e^{-\vartheta x} d x=\sum_{i, j=0}^{\infty} \xi_{i, j}^{(1+s, \vartheta t)} \gamma(1+s, \vartheta t)
$$

and

$$
\xi_{i, j}^{(1+s, \vartheta t)}=\frac{1}{(\vartheta t)^{1+s}} \xi_{i, j}
$$

$$
\left.\gamma(\tau, q)\right|_{(\tau \neq 0,-1,-2, \ldots)}=\int_{0}^{q} t^{\tau-1} e^{-t} d t=\frac{q^{\tau}}{\tau}\left\{1 F_{1}[\tau ; \tau a+1 ;-q]\right\}=\sum_{k=0}^{\infty} \frac{(-1)^{k}}{k !(\tau+k)} q^{\tau+k},
$$

is the lower incomplete gamma function. Similarly, the $\mathrm{r}$ th upper incomplete moment of McETEx distribution is

where

$$
\eta_{s}(t)=\int_{t}^{\infty} x^{s} f(x) d x=\sum_{i, j=0}^{\infty} \xi_{i, j} \int_{t}^{\infty} x^{s} e^{-\vartheta x} d x=\sum_{i, j=0}^{\infty} \xi_{i, j}^{(1+s, \vartheta t)} \Gamma(1+s, \vartheta t),
$$

$$
\left.\Gamma(\tau, q)\right|_{(z>0)}=\int_{0}^{q} t^{\tau-1} e^{-t} d t \sim \frac{q^{\tau-1}}{e^{q}}\left[1+\frac{\tau-1}{q}+\frac{(\tau-1)(\tau-2)}{q^{2}}+\ldots\right],
$$

is the upper incomplete gamma function, $1 F_{1}[\because, \cdot]$ is a confluent hypergeometric function and

$$
\Gamma(\tau, q)+\gamma(\tau, q)=\Gamma(\tau)
$$

The MRL has many applications in biomedical sciences, life insurance, maintenance and product quality control, economics and social studies, demography and product technology (see Lai and Xie, 2006). Guess and Proschan (1988) gave an extensive coverage of possible applications of the mean residual life. The MRL (or the life expectancy at age $t$ ) represents the expected additional life length for a unit, which is alive at age $t$. The MRL is given by

$$
m_{X}(t)=E\left(\left.X\right|_{(X>t)}\right)=\frac{\eta_{1}(t)}{\bar{F}(t)}-t,
$$

where $\eta_{1}(t)$ is the first incomplete moment of $X$ and by setting $s=1$ in equation (4.4), we get

$$
m_{X}(t)=\sum_{i, j=0}^{\infty} \frac{1}{\bar{F}(t)} \xi_{i, j}^{(2, \vartheta t)} \Gamma(2, \vartheta t)-t
$$

Also, the mean inactivity time (MIT) represents the waiting time elapsed since the failure of an item on condition that this failure had occurred in $(0 ; t)$. The MIT of $X$ is defined (for $t>0)$ by

$$
\tau_{X}(t)=E\left(\left.X\right|_{(X<t)}\right)=t-\frac{v_{1}(t)}{F(t)}=t-\frac{1}{F(t)} \xi_{i, j}^{(2, \vartheta t)} \gamma(2, \vartheta t)
$$

\section{Mean deviation, Lorenz and Bonferroni curves}

If $X$ has the McETEx distribution, then we can derive the mean deviations about the mean $\mu=E(X)$ and the mean deviations about the median $M$ are defined by

and

$$
\delta_{1}(x)=\int_{0}^{\infty} f(x)|x-\mu| d x=2[\mu F(\mu)-J(\mu)]
$$

$$
\delta_{2}(x)=\int_{0}^{\infty} f(x)|x-M| d x=\mu-2 J(M)
$$

respectively. The measures $\delta_{1}(x)$ and $\delta_{2}(x)$ can be calculated using the relationships

$$
J(d)=\int_{0}^{d} f(x) x d x=\sum_{i, j=0}^{\infty} \xi_{i, j}^{(2, \vartheta t)} \gamma(2, \vartheta d) .
$$

\section{Bonferroni and Lorenz Curves}


The Lorenz curve for a positive random variable $X$ is defined as

$$
L(p)=\frac{1}{\mu} \int_{0}^{q} f(x) x d x=\frac{1}{\mu} J(q)=\sum_{i, j=0}^{\infty} \frac{1}{\mu} \xi_{i, j}^{(2, \vartheta t)} \gamma(2, \vartheta d)
$$

where $q=G^{-1}(p)$. If $X$ represents annual income, $L(p)$ is the proportion of total income that accrues to individuals having the $100 \mathrm{p} \%$ lowest incomes. If all individuals earn the same income, then $L(p)=p$ for all $p$. The area between the line $L(p)=p$ and the Lorenz curve may be regarded as a measure of inequality of income. Also Bonferroni curve is defined by

$$
\begin{aligned}
& B(p)=\frac{1}{\mu p} \int_{0}^{q} f(x) x d x=\frac{1}{\mu p} J(q) \\
& =\sum_{i, j=0}^{\infty} \frac{1}{\mu} \xi_{i, j}^{(2, \vartheta t)} \gamma(2, \vartheta d),
\end{aligned}
$$

where the Bonferroni curve has many applications not only in economics to study income and poverty, but also in other fields like reliability, medicine and insurance.

\section{Residual life and reversed failure rate function}

Suppose that a component survives up to time $t \geq 0$, the residual life is the period beyond $t$ until the time of failure and defined by the conditional random variable $X-t \mid(X>t)$. Therefore, we obtain the $r^{t h}$ order moment of the residual life via the general formula

$$
z_{r}(t)=E\left((X-t)^{r} \mid X>t\right)=\left.\frac{1}{\bar{F}(t)} \int_{t}^{\infty} f(x)(x-t)^{r} d x\right|_{(r \geq 1)} .
$$

Applying the binomial expansion of $(x-t)^{r}$ and substituting $f_{\text {McETEx }}^{(\phi)}(x)$ given by (2.2) into the above formula gives

where

$$
z_{r}(t)=\sum_{i, j=0}^{\infty} \frac{\xi_{i, j}}{\bar{F}(t)} \sum_{m=0}^{r}(-t)^{m}\left(\begin{array}{c}
r \\
m
\end{array}\right) \int_{t}^{\infty} e^{-\vartheta x} x^{r-m} d x=\sum_{i, j=0}^{\infty} \sum_{m=0}^{r} \frac{\xi_{i, j, m}^{(1+r-m, \vartheta t)}}{\bar{F}(t)} \Gamma(1+r-m, \vartheta t)
$$

and

$$
\Gamma(s, t)=\int_{t}^{\infty} e^{-x} x^{s-1} d x
$$

$$
\xi_{i, j, m}^{(1+r-m, \vartheta t)}=\xi_{i, j}^{(1+r-m, \vartheta t)}(-t)^{m}\left(\begin{array}{c}
r \\
m
\end{array}\right)
$$

is the upper incomplete gamma function. The mean residual life (MRL) of the McETEx distribution is given by

$$
z(t)=\sum_{i, j=0}^{\infty} \frac{\xi_{i, j}^{(2, \vartheta t)}}{\bar{F}(t)} \Gamma(2, \vartheta t)-t
$$

The variance of the residual life of the McETEx distribution can be obtained easily by using $\mu_{2}(t)$ and $\mu(t)$.

Also, we analogously discuss the reversed residual life and some of its properties. The reversed residual life can be defined as the conditional random variable $t-X \mid X \leq t$ which denotes the time elapsed from the failure of a component given that its life is less than or equal to $t$. This random variable may also be called the inactivity time (or time since failure); for more details you may (see Kundu and Nanda, (2010)). Also, in reliability, the mean reversed residual life and ratio of two consecutive moments of reversed residual life characterize the distribution uniquely. The $r$ th order moment of the reversed residual life can be obtained by the well-known formula

$$
Z_{r}(t)=E\left(\left.(t-X)^{r}\right|_{(X \leq t)}\right)=\frac{1}{F(t)} \int_{0}^{t} f(x)(t-x)^{r} d x, r \geq 1 .
$$

Applying the binomial expansion of $(t-x)^{r}$ and substituting $f_{\text {McETEx }}^{(\phi)}(x)$ given by (4) into the above formula gives

$$
Z_{r}(t)=\sum_{m=0}^{r}\left(\begin{array}{c}
r \\
m
\end{array}\right)(-t)^{m} \int_{0}^{t} e^{-\vartheta x} x^{r-m} d x=\sum_{i, j=0}^{\infty} \sum_{m=0}^{r} \frac{1}{F(t)} \xi_{i, j}^{(1+r-m, \vartheta t)}(-t)^{m}\left(\begin{array}{c}
r \\
m
\end{array}\right) \gamma(1+r-m, \vartheta t)
$$

where 


$$
\gamma(s, t)=\int_{0}^{t} e^{-x} x^{s-1} d x
$$

is the lower incomplete gamma function. Thus, the mean reversed residual life (or mean waiting time) of the McETEx distribution is given by

$$
Z(t)=t-\sum_{i, j=0}^{\infty} \frac{1}{\bar{F}(t)} \xi_{i, j}^{(2, \vartheta t)} \gamma(2, \vartheta t) .
$$

Using $Z(t)$ and $Z_{2}(t)$ one can obtain the variance and the coefficient of variation of the reversed residual life of the McETEx distribution.

\section{Estimtion}

In this section, we determine the maximum likelihood estimates (MLEs) of the parameters of the McETEx distribution from complete samples only. Let $X_{1}, X_{2}, \ldots, X_{n}$ be a random sample of size $n$ from McETEx where $\mathbf{V}=$ $(\alpha, \theta, a, b, c)^{T}$ is the parameter vector. The log likelihood function for the vector of parameters $\mathbf{V}$ can be written easily derived and the components of the score vector as well.

\section{Application}

In this Section, we provide two applications to a real data sets to assess the flexibility of the McETEx model. Figure 2 gives the total time test (TTT) plots (see Aarset (1987)), box plots and Quantile-Quantile (Q-Q) plots for the two real data sets. Based on Figure 2 we note that the HRF of the two data are "increasing", the first data contains an extreme value, the second data contains four extreme values. The kernel density estimation (KDE) is a non-parametric way to estimate the probability density function of a random variable. KDE is a fundamental data smoothing problem where inferences about the population are made based on a finite data sample. Let $\left(w_{1}, w_{1}, \ldots, w_{n}\right)$ be a univariate independent and identically distributed sample drawn from some distribution with an unknown density function. Then, kernel density estimator is

$$
\hat{f}_{h}(w)=\frac{1}{n} \sum_{i=1}^{n} K_{h}\left(w-w_{i}\right)=\frac{1}{n h} \sum_{i=1}^{n} K\left(\frac{w-w_{i}}{h}\right),
$$

where $K$ is the non-negative kernel function, and $h>0$ is a smoothing parameter called the bandwidth. Figure 3 gives the KDE plots (see Rosenblat (1956) and Parzen (1962)). In order to compare the McETEx model with other fitted distributions, we compare the fits of the McETEx distribution with the Exponential $(\operatorname{Ex}(\theta))$, Odd Lindley Exponential (OLiEx), Marshall-Olkin Exponential (MOEx $(\alpha, \theta)$ ), Moment Exponential (MomEx $(\theta)$ ), The Logarithmic BurrHatke Exponential (Log $\operatorname{BrHEx}(\theta))$, Generalized Marshall-Olkin Exponential (GMOEx $(\lambda, \alpha, \theta))$, Beta Exponential (BEx $(a, b, \theta))$, Burr X Ex $(a, \theta)$, Marshall-Olkin Kumaraswamy Exponential (MOKEx $(\alpha, a, b, \theta)$ ), Kumaraswamy Exponential (KEx $(a, b, \theta))$ and Kumaraswamy Marshall-Olkin Exponential (KMOEx $(\alpha, a, b, \theta))$ models. The first data set $\{1.1,1.4,1.3,1.7,1.9,1.8,1.6,2.2,1.7,2.7,4.1,1.8,1.5,1.2,1.4,3,1.7,2.3,1.6,2\}$ called the failure time data: The data represents the lifetime data relating to relief times (in minutes) of patients receiving an analgesic (see Gross and Clark (1975) ). The second data set $\{0.1,0.33,0.44,0.56,0.59,0.72,0.74,0.77,0.92,0.93,0.96,1,1,1.02$, $1.05,1.07,07,1.08,1.08,1.08,1.09,1.12,1.13,1.15,1.16,1.2,1.21,1.22,1.22,1.24,1.3,1.34,1.36,1.39,1.44,1.46$, $1.53,1.59,1.6,1.63,1.63,1.68,1.71,1.72,1.76,1.83,1.95,1.96,1.97,2.02,2.13,2.15,2.16,2.22,2.3,2.31,2.4$, $2.45,2.51,2.53,2.54,2.54,2.78,2.93,3.27,3.42,3.47,3.61,4.02,4.32,4.58,5.55\}$ called the survival times (in days) of 72 guinea pigs infected with virulent tubercle bacilli, observed and reported by Bjerkedal (1960). 

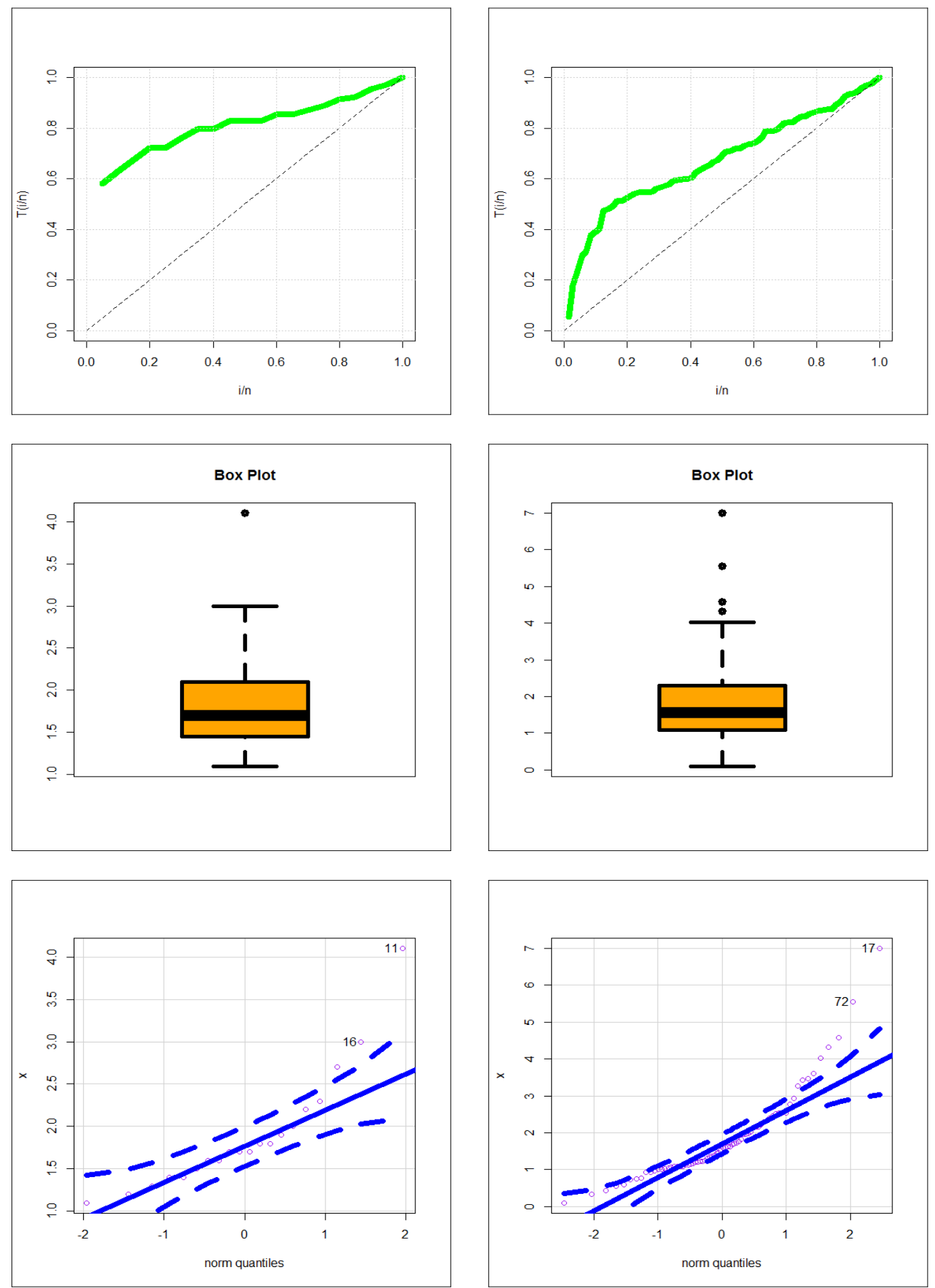

First data set

Second data set

Figure 2: TTT plots, box plots and Q-Q plots. 


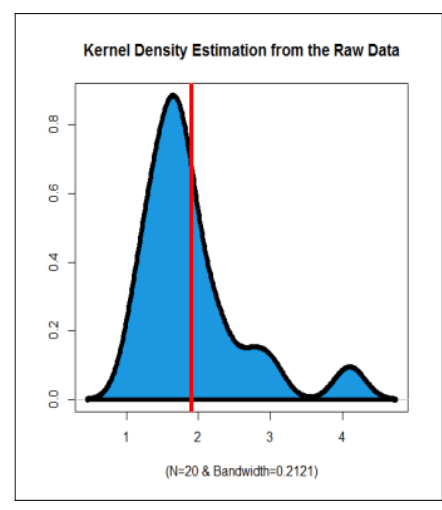

First data set

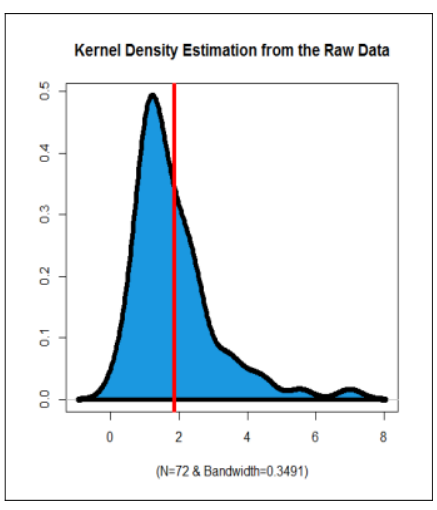

Second data set

Figure 3: Kernel density estimation

We consider the Cramér-Von Mises and the Anderson-Darling $\left(A^{*}, W^{*}\right)$ and the Kolmogorov-Smirnov (KS) statistic. Moreover, we consider some other goodness-of-fit measures including the Akaike Information Criterion, Consistent Akaike Information Criterion, Hannan-Quinn Information Criterion and Bayesian Information Criterion $\left(\mathrm{C}_{1}, \mathrm{C}_{2}, \mathrm{C}_{3}\right.$ and $\mathrm{C}_{4}$ ). Tables 2 and 4 gives the MLEs and SEsvalues for the two data sets. Table 3 and 5 gives the $\mathrm{C}_{1}, \mathrm{C}_{2}, \mathrm{C}_{3}$, $\mathrm{C}_{4}, A^{*}, W^{*}, \mathrm{~K} . \mathrm{S}$. and (p-value) for the two data sets. Figures 4 and 5 gives the estimated PDF (ECDF), EHRF, P-P plot and Kaplan-Meier Survival Plot for the two data sets.
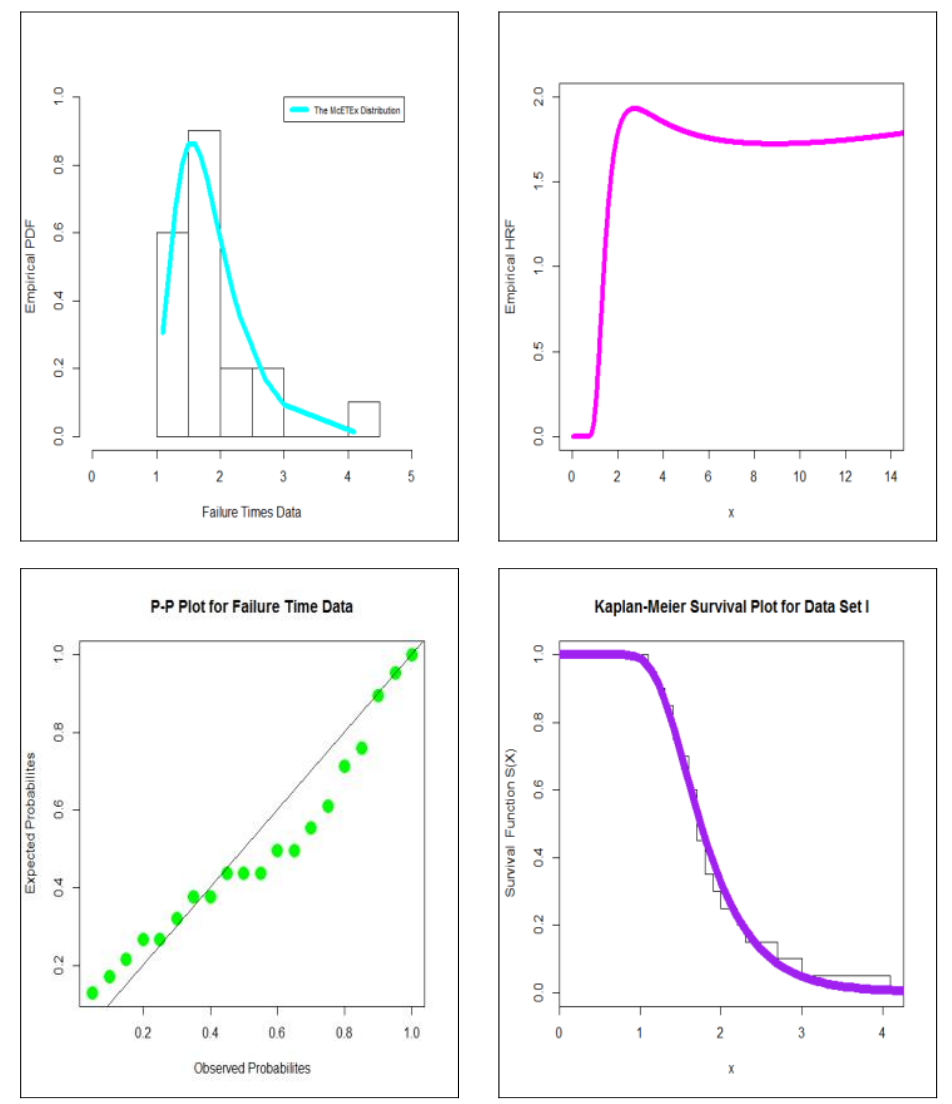

Figure 4: EPDF, EHRF, P-P plot and Kaplan-Meier Survival Plot for the 1st data. 

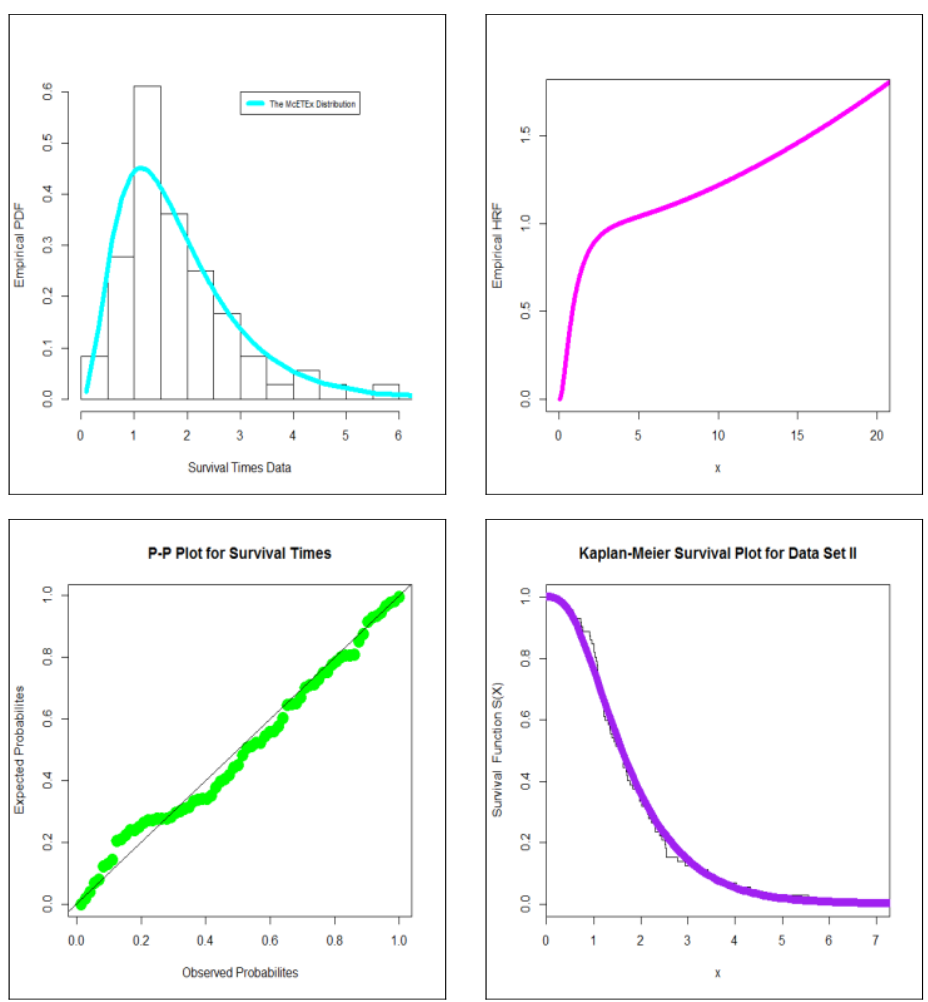

Figure 5: EPDF, EHRF, P-P plot and Kaplan-Meier Survival Plot for the 2nd data.

Table 2: MLEs and SEs values for the relief times data.

\begin{tabular}{|c|c|c|}
\hline Models & & Estimates \\
\hline \multirow[t]{2}{*}{$\operatorname{Ex}(\theta)$} & MLE & 0.526 \\
\hline & $\mathrm{SE}$ & $(0.117)$ \\
\hline \multirow[t]{2}{*}{$\operatorname{OLEx}(\theta)$} & MLE & 0.6044 \\
\hline & SE & $(0.0535)$ \\
\hline \multirow[t]{2}{*}{$\operatorname{MEx}(\theta)$} & MLE & 0.950 \\
\hline & SE & $(0.150)$ \\
\hline \multirow[t]{2}{*}{$\operatorname{LBHEx}(\theta)$} & MLE & 0.5263 \\
\hline & SE & $(0.118)$ \\
\hline \multirow[t]{2}{*}{$\operatorname{MOEx}(\alpha, \theta)$} & MLE & $54.474,2.316$ \\
\hline & SE & $(35.582),(0.374)$ \\
\hline \multirow[t]{2}{*}{$\operatorname{GMOEx}(\lambda, \alpha, \theta)$} & MLE & $0.519,89.462,3.169$ \\
\hline & SE & $(0.256),(66.278),(0.772)$ \\
\hline \multirow[t]{2}{*}{$\operatorname{KwEx}(\alpha, \beta, \theta)$} & MLE & $83.756,0.568,3.330$ \\
\hline & SE & (42.361), (0.326), (1.188) \\
\hline \multirow[t]{2}{*}{$\operatorname{BEx}(\alpha, \beta, \theta)$} & MLE & $81.633,0.542,3.514$ \\
\hline & SE & $(120.41),(0.327),(1.410)$ \\
\hline \multirow[t]{2}{*}{$\operatorname{MOKwEx}(\alpha, \beta, \lambda, \theta)$} & MLE & $0.133,33.232,0.571,1.669$ \\
\hline & SE & $(0.332),(57.837),(0.721),(1.814)$ \\
\hline \multirow[t]{2}{*}{$\operatorname{KwMOEx}(\alpha, \beta, \lambda, \theta)$} & MLE & $8.868,34.826,0.299,4.899$ \\
\hline & SE & (9.146), (22.312), (0.239), (3.176) \\
\hline \multirow[t]{2}{*}{$\operatorname{BrXEx}(\mathrm{a}, \theta)$} & MLE & $1.1635,0.3207$ \\
\hline & SE & $(0.33),(0.03)$ \\
\hline \multirow[t]{2}{*}{$\operatorname{McETEx}(\mathbf{a}, \mathbf{b}, \mathbf{c}, \alpha, \theta)$} & MLE & $5.307,0.866,6.052,188.66,0.012$ \\
\hline & SE & $(2.201),(0.515),(0.266),(7.458),(0.118)$ \\
\hline
\end{tabular}


Table 3: $\mathrm{C}_{1}, \mathrm{C}_{2}, \mathrm{C}_{3}, \mathrm{C}_{4}, A^{*}, C^{*}, \mathrm{~K} . \mathrm{S}$. and (p-value) for the relief times data.

\begin{tabular}{|c|c|c|c|}
\hline Models & $\mathrm{C}_{1}, \mathrm{C}_{2}, \mathrm{C}_{3}, \mathrm{C}_{4}$ & $A^{*}, C^{*}$ & $\begin{array}{c}\text { K.S. } \\
\text { (p-value) }\end{array}$ \\
\hline McETEx & $42.46,38.96,46.74,43.43$ & $0.32,0.05$ & $0.11(0.96)$ \\
\hline BEx & $43.48,46.45,44.98,44.02$ & $0.70,0.12$ & $0.16(0.80)$ \\
\hline Ex & $67.67,68.67,67.89,67.87$ & $4.60,0.96$ & $0.44(0.004)$ \\
\hline BrXEx & $48.13,50.51,48.83,48.54$ & $1.39,0.24$ & $0.3(0.171)$ \\
\hline OLEx & $49.14,50.13,49.34,49.35$ & $1.35,0.22$ & $0.9(<0.001)$ \\
\hline KwEx & $41.78,44.75,43.28,42.32$ & $0.45,0.07$ & $0.14(0.86)$ \\
\hline MOKEx & $41.58,45.54,44.25,42.30$ & $0.60,0.11$ & $0.14(0.87)$ \\
\hline LBHEx & $67.67,68.67,67.89,67.87$ & $0.62,0.11$ & $0.5(<0.001)$ \\
\hline MEx & $54.32,55.31,54.54,54.50$ & $2.76,0.53$ & $0.32(0.07)$ \\
\hline MOEx & $43.51,45.51,44.22,43.90$ & $0.82,0.14$ & $0.18(0.55)$ \\
\hline GMOEx & $42.75,45.74,44.25,43.34$ & $0.51,0.08$ & $0.15(0.78)$ \\
\hline KMOEx & $42.82,46.84,45.55,43.60$ & $1.08,0.19$ & $0.15(0.86)$ \\
\hline
\end{tabular}

Table 4: MLEs and SEs values for the survival times data.

\begin{tabular}{|c|c|c|}
\hline Models & & Estimates \\
\hline \multirow[t]{2}{*}{$\operatorname{Ex}(\theta)$} & MLE & 0.540 \\
\hline & SE & $(0.063)$ \\
\hline \multirow[t]{2}{*}{$\operatorname{OLEx}(\theta)$} & MLE & 0.38145 \\
\hline & SE & $(0.0209)$ \\
\hline \multirow[t]{2}{*}{$\operatorname{MEx}(\theta)$} & MLE & 0.925 \\
\hline & SE & $(0.077)$ \\
\hline \multirow[t]{2}{*}{$\operatorname{LBHEx}(\theta)$} & MLE & 0.54 \\
\hline & SE & $(0.064)$ \\
\hline \multirow[t]{2}{*}{$\operatorname{MOEx}(\alpha, \theta)$} & MLE & $8.778,1.379$ \\
\hline & $\mathrm{SE}$ & $(3.555),(0.193)$ \\
\hline \multirow[t]{2}{*}{$\operatorname{GMOEx}(\lambda, \alpha, \theta)$} & MLE & $0.179,47.635,4.465$ \\
\hline & $\mathrm{SE}$ & $(0.070),(44.901),(1.327)$ \\
\hline \multirow[t]{2}{*}{$\operatorname{KwEx}(\mathrm{a}, \beta, \theta)$} & MLE & $3.304,1.100,1.037$ \\
\hline & $\mathrm{SE}$ & $(1.106),(0.764),(0.614)$ \\
\hline \multirow[t]{2}{*}{$\operatorname{BEx}(\mathrm{a}, \beta, \theta)$} & MLE & $0.807,3.461,1.331$ \\
\hline & $\mathrm{SE}$ & $(0.696),(1.003),(0.855)$ \\
\hline \multirow[t]{2}{*}{$\operatorname{MOKEx}(\alpha, \beta, \lambda, \theta)$} & MLE & $0.008,2.716,1.986,0.099$ \\
\hline & $\mathrm{SE}$ & $(0.002), 1.316),(0.784),(0.048)$ \\
\hline \multirow[t]{2}{*}{$\operatorname{KwMOEx}(\alpha, \beta, \lambda, \theta)$} & MLE & $0.373,3.478,3.306,0.299$ \\
\hline & $\mathrm{SE}$ & $(0.136),(0.861),(0.779),(1.112)$ \\
\hline \multirow[t]{2}{*}{$\operatorname{BrXEx}(\mathrm{a}, \theta)$} & MLE & $0.475,0.2055$ \\
\hline & SE & $(0.06),(0.012)$ \\
\hline \multirow[t]{2}{*}{$\operatorname{McETEx}(\mathbf{a}, \mathbf{b}, \mathbf{c}, \boldsymbol{\alpha}, \theta)$} & MLE & $1.88,3.311,0.909,19.415,3.87$ \\
\hline & SE & $(0.918),(0.905),(4.801),(2.092),(1.948)$ \\
\hline
\end{tabular}


Table 5: $\mathrm{C}_{1}, \mathrm{C}_{2}, \mathrm{C}_{3}, \mathrm{C}_{4}, A^{*}, C^{*}, \mathrm{~K} . \mathrm{S}$. and (p-value) for the survival times data.

\begin{tabular}{cccc}
\hline Models & $\mathrm{C}_{1}, \mathrm{C}_{2}, \mathrm{C}_{3}, \mathrm{C}_{4}$ & $A^{*}, C^{*}$ & $\begin{array}{c}\text { K.S. } \\
(\mathrm{p} \text {-value })\end{array}$ \\
\hline McETEx & $\mathbf{2 0 5 . 8 1 , 2 1 2 . 6 2 , 2 0 5 . 1 1 , 2 0 7 . 8 8}$ & $\mathbf{0 . 5 8 , 0 . 0 9}$ & $\mathbf{0 . 0 9}(\mathbf{0 . 5 9})$ \\
Ex & $234.63,236.91,234.68,235.54$ & $6.53,1.25$ & $0.27(0.06)$ \\
BEx & $207.38,214.22,207.73,210.08$ & $0.98,0.15$ & $0.11(0.34)$ \\
BrXEx & $235.30,239.90,235.50,237.11$ & $2.91,0.52$ & $0.22(0.002)$ \\
MEx & $210.40,212.68,210.45,211.30$ & $1.52,0.25$ & $0.14(0.13)$ \\
LBHEx & $234.63,236.92,234.73,235.55$ & $0.71,0.12$ & $0.28(<0.001)$ \\
KMOEx & $207.82,216.94,208.42,211.42$ & $0.61,0.11$ & $0.09(0.53)$ \\
OLEx & $229.31,231.43,229.25,230.00$ & $1.94,0.33$ & $0.5(<0.001)$ \\
GMOEx & $210.54,217.38,210.89,213.24$ & $1.02,0.16$ & $0.09(0.51)$ \\
MOKEx & $209.44,218.56,210.04,213.04$ & $0.79,0.12$ & $0.10(0.44)$ \\
MOEx & $210.36,214.92,210.53,212.16$ & $1.18,0.17$ & $0.10(0.43)$ \\
KwEx & $209.42,216.24,209.77,212.12$ & $0.74,0.11$ & $0.09(0.50)$ \\
\hline
\end{tabular}

From Table 3 and 5 we conclude that the proposed lifetime McETEx model is much better than the Ex, MomEx, MOEx, GMOEx, KEx, BEx, MOKEx and KMOEx models so the new lifetime model is a good alternative to these models in modeling relief times and survival times data sets.

\section{Conclusions}

In this article, we introduced a new distribution called the McDonald Erlang-truncated exponential (McETEx) distribution. Various structural properties including explicit expressions for the moments, moment generating function, mean deviation of the McETEx distribution are derived. The estimation of the model parameters is performed by maximum likelihood method. The usefulness of the McETEx distribution is illustrated by two real data sets. The McETEx model is much better than the Exponential, Odd Lindley Exponential, Marshall-Olkin Exponential, Moment Exponential, The Logarithmic Burr-Hatke Exponential, Generalized Marshall-Olkin Exponential, Beta Exponential, Burr X Exponential, Marshall-Olkin Kumaraswamy Exponential, Kumaraswamy Exponential and Kumaraswamy Marshall-Olkin Exponential models. in modeling relief times and survival times data sets according to Cramér-Von Mises, the Anderson-Darling, Akaike Information Criterion, Consistent Akaike Information Criterion, Hannan-Quinn Information Criterion, Bayesian Information Criterion.

\section{Acknowledgements}

The authors acknowledge the Deanship of Scientific Research at Al Imam Mohammad Ibn Saud Islamic UniversitySaudi Arabia, for financing this project under the grant no (381207).

\section{References}

1. Aarset, M. V. (1987). How to identify a bathtub hazard rate. IEEE Transactions on Reliability, 36(1), 106-108.

2. Bjerkedal, T. (1960). Acquisition of resistance in Guinea pigs infected with different doses of virulent tubercle bacilli. American Journal of Hygiene, 72, 130-148.

3. Alizadeh, M., Jamal, F., Yousof, H. M., Khanahmadi, M. and Hamedani, G. G. (2020). Flexible Weibull generated family of distributions: characterizations, mathematical properties and applications. University Politehnica of Bucharest Scientific Bulletin-Series A- Applied Mathematics and Physics, 82(1), 145-150.

4. Alizadeh, M., Lak, F., Rasekhi, M., Ramires, T. G., Yousof, H. M. and Altun, E. (2018). The odd log-logistic Topp-Leone $\mathrm{G}$ family of distributions: heteroscedastic regression models and applications. Computational Statistics, 33(3), 1217-1244.

5. Cordeiro, G. M. and Castro, M. (2011). A new family of generalized distributions. Journal of Statistical Computation and Simulation. 81(7), 883-898.

6. Cordeiro, G. M. Cintra, R. J., Rêgo, L. C. and Ortega, E. M. M. (2012). The McDonald normal distribution. Pakistan Journal of Statistics and Operation Research, 8(3), 301-329.

7. El-Alosey,A. R (2007). Random sum of new type of mixture of distribution, International Journal of Statistics and Systems, 2, 49-57.

8. Elbiely, M. M. and Yousof, H. M. (2019). A new flexible Weibull Burr XII distribution. Journal of Statistics and Applications, 2(1), 59-77. 
9. Eugene, N., Lee, C., Famoye, F. (2002). Beta-normal distribution and its applications. Communications in Statistics-Theory and Methods 31(4), 497-512.

10. Gradshteyn, I. S. and Ryzhik, I. M. (2000). Table of Integrals, Series, and Products (sixth edition). San Diego: Academic Press.

11. Gross, J. and Clark, V. A. (1975). Survival Distributions: Reliability Applications in the Biometrical Sciences, John Wiley, New York, USA

12. Guess, F. and Proschan, F. (1988). Mean residual life: theory and applications. In Handbook of Statistics (P. R. Krishnaiah and C. R. Rao, eds.) 7 215-224. North-Holland, New York

13. Gupta, R. C. (1981) On the mean residual life function in survival studies, Distributions in Scientific Work 5, DReidel Publishing Co. Boston, 327-334.

14. Hamedani, G. G., Altun, E., Korkmaz, M. Ç., Yousof, H. M., \& Butt, N. S. (2018). A new extended G family of continuous distributions with mathematical properties, characterizations and regression modeling. Pakistan Journal of Statistics and Operation Research, 737-758.

15. Ibrahim, M. and Yousof, H. (2020). Transmuted Topp-Leone Weibull lifetime distribution: Statistical properties and different method of estimation. Pakistan Journal of Statistics and Operation Research, 501-515.

16. Ibrahim, M., Altun, E. and Yousof, H. M. (2020). A new distribution for modeling lifetime data with different methods of estimation and censored regression modeling. Statistics, Optimization \& Information Computing, 8(2), 610-630.

17. Karamikabir, H., Afshari, M., Yousof, H. M., Alizadeh, M., \& Hamedani, G. (2020). The Weibull Topp-Leone Generated Family of Distributions: Statistical Properties and Applications. Journal of The Iranian Statistical Society, 19(1), 121-161.

18. Korkmaz, M. Ç., Alizadeh, M., Yousof, H. M. and Butt, N. S. (2018). The generalized odd Weibull generated family of distributions: statistical properties and applications. Pakistan Journal of Statistics and Operation Research, 541-556.

19. Korkmaz, M. Ç., Altun, E., Yousof, H. M. and Hamedani, G. G. (2019a). The odd power Lindley generator of probability distributions: properties, characterizations and regression modeling. International Journal of Statistics and Probability. 8(2), 70-89.

20. Korkmaz, M. Ç., Altun, E., Yousof, H. M. and Hamedani, G. G. (2020). The Hjorth's IDB Generator of Distributions: Properties, Characterizations, Regression Modeling and Applications. Journal of Statistical Theory and Applications, 19(1), 59-74.

21. Korkmaz, M. Ç., Cordeiro, G. M., Yousof, H. M., Pescim, R. R., Afify, A. Z. and Nadarajah, S. (2019b). The Weibull Marshall-Olkin family: Regression model and application to censored data. Communications in Statistics-Theory and Methods, 48(16), 4171-4194.

22. Korkmaz, M. C., Yousof, H. M., Alizadeh, M. and Hamedani, G. G. (2019c). The Topp-Leone generalized odd log-logistic family of distributions: properties, characterizations and applications. Communications Faculty of Sciences University of Ankara Series A1 Mathematics and Statistics, 68(2), 1506-1527.

23. Kundu, D., Nanda, A.K., 2010. Some reliability properties of the inactivity time. Communications in StatisticsTheory and Methods 39, 899-911.

24. Lai, C. D and Xie, M (2006). Stochastic Ageing and Dependence for Reliability. Springer, New York.

25. Marciano, F. W. P., Nascimento, A. D. C., Santos-Neto, M., and Cordeiro, G. M. (2012). The Mc- $\Gamma$ distribution and its statistical properties: An application to reliability data. International Journal of Statistics and Probability, 1(1), 53-71.

26. Mi, J (1996). Limiting Behavior of Mixtures of Discrete Lifetime Distributions. Naval Researc Logistics, 43, 365380.

27. Nasiru, S., Luguterah, A. and Iddrisu, M.M. (2016). Generalized Erlang-truncated exponential distribution, Adv. Appl. Stat. 48(4), 273-301.

28. Okorie, I. E., Akpanta A. C. and Ohakwe, J. (2017). Marshall-Olkin generalized Erlang-truncated exponential distribution: Properties and applications. Cogent Mathematics, 4, 1-19.

29. Okorie, I. E., Akpanta A. C. and Ohakwe, J. (2016). Transmuted Erlang-truncated exponential distribution, Econ. Qual. Control 31(2), 71-84.

30. Oliveira, J., Santos, J., Xavier, C., Trindade, D., and Cordeiro, G. M. (2013). The McDonald half-logistic distribution: Theory and practice. Communications in Statistics-Theory and Methods, 10.1080/03610926.2013.873131.

31. Oluyede, B. O. and Rajasooriya, S. (2013). The Mc-Dagum distribution and its statistical properties with applications. Asian Journal of Mathematics and Applications, 2013, 1-16.

32. Parzen, E. (1962). On Estimation of a Probability Density Function and Mode. The Annals of Mathematical 
Statistics. 33 (3), 1065-1076.

33. Roozegar, R., Tahmasebi, S. and Jafari, A. A. (2017). The McDonald Gompertz distribution: properties and applications, 47(5), 3341-3355.

34. Rosenblatt, M. (1956). Remarks on Some Nonparametric Estimates of a Density Function. The Annals of Mathematical Statistics. 27 (3), 832-837. doi:10.1214/aoms/1177728190

35. Yousof, H. M., Altun, E., Rasekhi, M., Alizadeh, M., Hamedani, G. G. and Ali, M. M. (2019). A new lifetime model with regression models, characterizations and applications. Communications in Statistics-Simulation and Computation, 48(1), 264-286.

36. Yousof, H. M., Majumder, M., Jahanshahi, S. M. A., Masoom Ali, M. and Hamedani, G. G. (2018a). A new Weibull class of distributions: theory, characterizations and applications. Journal of Statistical Research of Iran JSRI, 15(1), 45-82.

37. Yousof, H., Mansoor, M., Alizadeh, M., Afify, A. and Ghosh, I. (2020). The Weibull-G Poisson family for analyzing lifetime data. Pakistan Journal of Statistics and Operation Research, 131-148.

38. Yousof, H. M., Rasekhi, M., Alizadeh, M. and Hamedani, G. G. (2018b). The Marshall-Olkin exponentiated generalized $\mathrm{G}$ family of distributions: properties, applications and characterizations. Journal of Nonlinear Sciences and Applications, 13(1), 34-52

39. Yousof, H. M., Rasekhi, M., Altun, E. and Alizadeh, M. (2018c). The extended odd Frechet family of distributions: properties, applications and regression modeling. International Journal of Applied Mathematics and Statistics, 30(1), 1-30. 